\title{
$\Omega$
}

(1)

'Dept of Respiratory Medicine, King's College Hospital, London, UK.

2Dept of Thoracic Surgery, King's College Hospital, London, UK.

${ }^{3}$ Dept of Respiratory Medicine and Allergy, King's College London, London, UK.

\section{An interesting case of recurrent shortness of breath and pleuritic chest pain}

\section{Case report}

\section{Case presentation}

A 58-year-old male presented to the emergency department in August 2015 with suddenonset shortness of breath associated with sharp right-sided pleuritic chest pain. His only past medical history was of hypertension. Prior to this presentation he had been clinically well and was fully independent. He was a smoker with a 40 packyear history. He did not drink alcohol and had no recent travel history.

On clinical examination the patient was unwell with a pain score of nine out of 10 . His heart rate was 76 beats per min, his blood pressure was $210 / 100 \mathrm{mmHg}$, his respiratory rate was
20 breaths per min and his oxygen saturations were $98 \%$ on room air.

His chest examination showed reduced air entry on the right side with reduced percussion note. He had poor chest expansion on the right side. Heart sounds were normal, the apex beat was in the midclavicular line and jugular venous pressure was normal. He had no peripheral oedema.

\section{Investigations}

\section{Task 1}

What would be your initial investigations in this case?
Cite as: Price K, Hall J, Nicou N, et al. An interesting case of recurrent shortness of breath and pleuritic chest pain. Breathe 2018; 14:123-130. 


\section{Answer 1}

Blood tests including inflammatory markers, haemoglobin, renal function and clotting.

These showed a C-reactive protein (CRP) of $48 \mathrm{mg} \cdot \mathrm{L}^{-1}$ (normal range: $<5 \mathrm{mg} \cdot \mathrm{L}^{-1}$ ) and white cell count (WCC) of $6.05 \times 10^{9} \mathrm{~L}^{-1}$ (normal range: 4-11 $\left.\times 10^{9} \mathrm{~L}^{-1}\right)$, with normal haemoglobin, platelets and renal function. A posterior to anterior chest radiograph was performed.

\section{Task 2}

What are the abnormalities in his chest radiograph (figure 1 )?

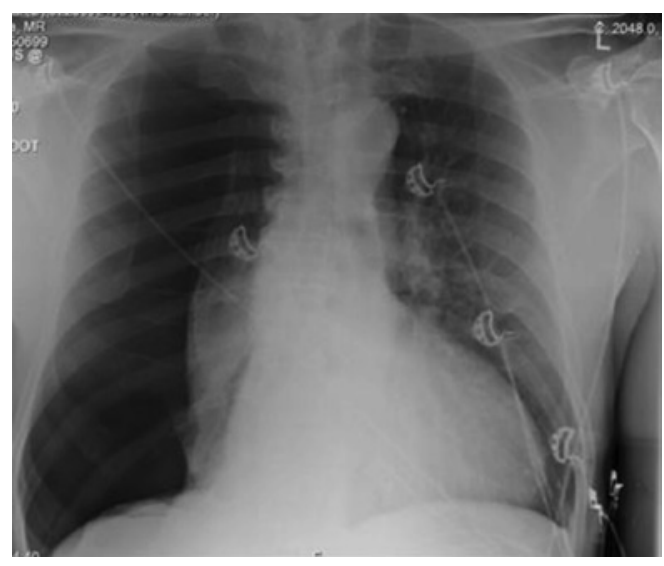

Figure 1 Chest radiograph.

\section{Answer 2}

A right-sided pneumothorax with cardiac monitoring attached. There is also background emphysema shown in the left lung.

He was diagnosed with a large right-sided spontaneous pneumothorax with possible background emphysematous disease warranting further investigation given the patient's smoking history.

\section{Treatment}

Task 3

How should this pneumothorax be managed and what are the factors which influence your decision? 


\section{Answer 3}

The management of pneumothoraces is determined by the underlying aetiology. Severe symptoms and signs of respiratory distress are indicative of a tension pneumothorax, which requires immediate decompression. Primary spontaneous pneumothorax is diagnosed in patients with no underlying lung disease and can be managed conservatively; however, in the presence of worsening breathlessness it is advised to consider intervention.

Secondary pneumothorax occurs in patients with underlying lung disease, which has occurred in this case given his smoking history and the underlying emphysematous lung disease shown on the chest radiograph. The British Thoracic Society guidelines recommend immediate chest drain insertion [1].

The British Thoracic Society algorithm for the management of spontaneous pneumothorax states that in primary pneumothorax $(>2 \mathrm{~cm})$ a chest drain should only be inserted following failed aspiration [1]. However, in secondary pneumothorax if patients are symptomatic $(>2 \mathrm{~cm})$ a chest drain should be inserted.

A size 12 Seldinger chest drain was inserted in the emergency department. The chest drain was working well, with bubbling initially. A repeat chest radiograph showed improvement of the pneumothorax size; however, following a number of days there was persistent breathlessness and bubbling of the chest drain despite the use of suction.

\section{Task 4}

When should thoracic surgical advice be sought in non-resolving pneumothorax?

\section{Answer 4}

Patients with a spontaneous secondary pneumothorax with a persistent air leak at $48 \mathrm{~h}$ should be discussed with a thoracic surgeon [1].

He was assessed by the cardiothoracic surgeons, with radiographs which confirmed persistent pneumothorax, who performed a right-sided videoassisted thoroscopic surgery (VATS) with apical bullectomy and abrasion pleurodesis. Although a chest computed tomography (CT) scan can be useful for patients with persistent pneumothorax in order to identify loculated pneumothoraces and secondary causes, it was not performed and the patient was offered surgery to treat the persistent air leak and reduce the risk of recurrent episodes. Postoperative recovery was uneventful and the patient was discharged on the forth post-operative day. At a follow-up appointment the patient had recovered back to his baseline [1]. He was assessed in the community and diagnosed with emphysematous chronic obstructive pulmonary disease (COPD).

\section{Re-presentation to the emergency department}

In July 2016, he re-presented to the emergency department a with sudden onset right-sided pleuritic chest pain. On this admission he was apyrexial, had a respiratory rate of 20 breaths per min, oxygen saturations of $98 \%$ on room air, normal blood pressure and a normal heart rate. On admission his CRP was $<2 \mathrm{mg} \cdot \mathrm{L}^{-1}$ (normal range: $<5 \mathrm{mg} \cdot \mathrm{L}^{-1}$ ) and his WCC was $5.8 \times 10^{9} \mathrm{~L}^{-1}$ (normal range: $4-11 \times 10^{9} \mathrm{~L}^{-1}$ ). On examination he had reduced air entry on the right side. A repeat chest radiograph showed a small right recurrent pneumothorax. ACT scan was carried out to assess this further.

\section{Task 5}

Please interpret the CT scan (figure 2): what does it show?

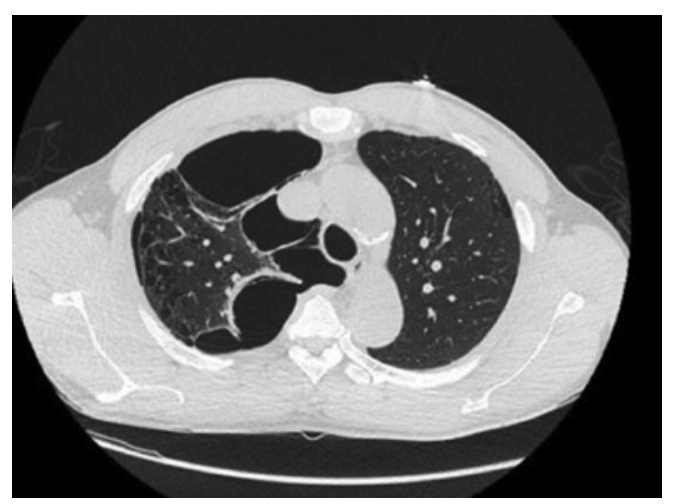

Figure 2 Chest CT scan, July 2016. 


\section{Answer 5}

Right-sided small pneumothorax with underlying large bullous disease.

He was admitted under the care of the thoracic surgeons and underwent a right thoracotomy, apical bullectomy and talc pleurodesis for recurrent pneumothorax. Post-operatively he presented with persistent air leak and the drains were kept in situ. 12 days following the procedure he became clinically unwell with spiking temperatures $\left(38.2^{\circ} \mathrm{C}\right)$ while his inflammatory markers increased to a CRP of $269 \mathrm{mg} \cdot \mathrm{L}^{-1}$ and a WCC $13.2 \times 10^{9} \mathrm{~L}^{-1}$. A repeat chest radiograph was performed (figure 3 ).

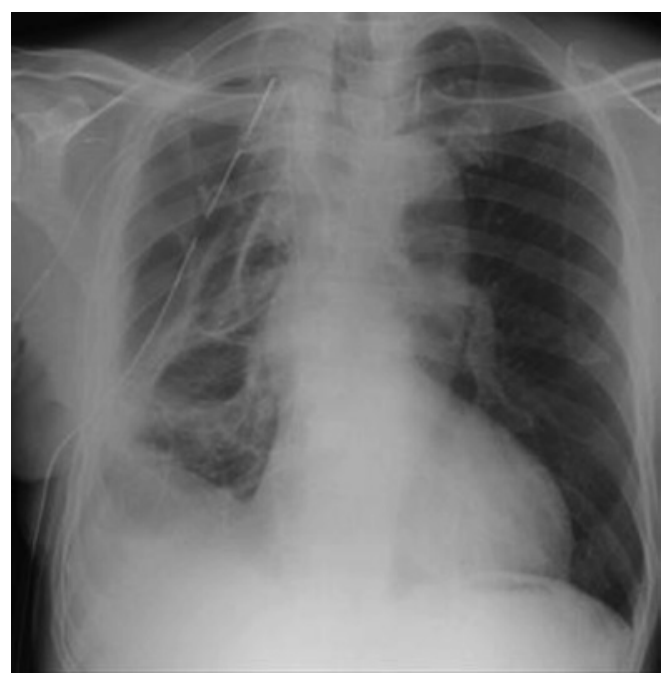

Figure 3 Chest radiograph following deterioration.

Task 6

What is the most likely diagnosis for this presentation?

\section{Answer 6}

Empyema, pneumonia with para-pneumonic effusion, haemothorax.

\section{Treatment}

Pleural fluid appeared turbid and pleural fluid cultures grew Proteus and Citrobacter. He was treated with a prolonged course of antibiotics, initially 14 days of intravenous antibiotics (Tazocin), but he continued to spike temperatures. 1 month into his admission he underwent repeat thoracotomy washout and decortication for empyema. Following surgery there was persistent air leak and a chest radiograph showed residual hydropneumothorax. At this stage it was deemed that further surgery would not be beneficial to the patient's management. The patient continued on intravenous antibiotics while the chest drains remained in situ.

\section{Task 7}

What options are available for surgically inoperable pneumothorax? 


\section{Answer 7} to guide management; primarily it should be ensured that the leak is from the lungs not the tubing system. Secondly, bronchopulmonary fistulas should be ruled out using imaging [2]. There are a number of options for treating persistent air leaks in patients with secondary spontaneous pneumothorax. Medical pleurodesis can be used, but has limited success so is restricted to those unable to tolerate surgical options [2]. An alternative to talc pleurodesis is autologous blood patch; this is considered effective and safe, particularly for those with advanced COPD who would not tolerate surgery [3]. Finally, Heimlich valves, a one-way valve attached to the chest tube which allows air or fluid to leave the chest cavity but not enter, are used in patients who would benefit from ambulatory care [4].
The location of the leak should be considered

rate was 75 beats per min, blood pressure was $117 / 63 \mathrm{mmHg}$, respiratory rate was 20 breaths per min and his oxygen saturations were $99 \%$ on room air. He was cachexic and had clubbing of his nails. Cardiovascular examination was normal. Examination of his respiratory system revealed reduced air entry in the right lung with dullness on percussion.

\section{Investigations}

Results of admission laboratory blood test investigations are shown in table 1.

The patient had a repeat chest CT due to the previous history of recurrent spontaneous pneumothoraces and hydropneumothorax.

\section{Task 8}

What can you see in this chest CT scan

(figure 4)?

Table 1 Admission laboratory blood test investigations was not a candidate for pleurodesis. Therefore, he was managed with a Heimlich valve device with successful resolution of his hydropneumothorax. He clinically improved and was discharged with a plan for review in the outpatient clinic.

\section{Re-presentation to the emergency department}

In July 2017, he re-presented to accident and emergency with progressive shortness of breath and a productive cough with green sputum. He denied history of malignancy, contact with tuberculosis or recent travel.

On clinical examination the patient was clinically unwell. His temperature was $36.2^{\circ} \mathrm{C}$, his heart

\begin{tabular}{lcc}
\hline Investigation & Patient value & Normal range \\
\hline Sodium mmol. $\mathrm{L}^{-1}$ & 138 & $135-145$ \\
Potassium mmol. $\mathrm{L}^{-1}$ & 4.8 & $3.5-5.0$ \\
Creatinine $\mu \mathrm{mol} \cdot \mathrm{L}^{-1}$ & 72 & $45-120$ \\
$\mathrm{CRP} \mathrm{mg} \cdot \mathrm{L}^{-1}$ & 99 & $<5$ \\
White cell count L-1 & $8.3 \times 10^{9}$ & $4-11 \times 10^{9}$ \\
Haemoglobin $\mathrm{g} \cdot \mathrm{L}^{-1}$ & 95 & $130-165$ \\
Platelets L-1 & $549 \times 10^{9}$ & $150-450 \times 10^{9}$ \\
INR & 1.09 & $0.9-1.20$ \\
INR: international normalised ratio. & &
\end{tabular}
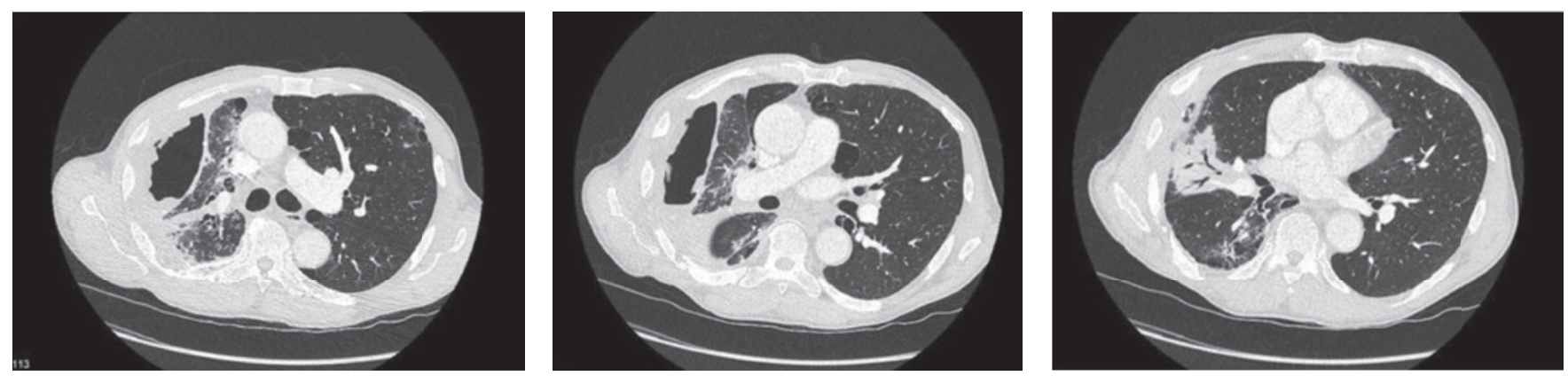

Figure 4 CT of the chest with multiple slices to review. 


\section{Answer 8}

The official radiological report for the chest CT showed:

- Volume loss in the right hemithorax with evidence of previous surgery at the right apex.

- Fairly large biconvex loculated hydropneumothorax anterolaterally in the right upper and mid zones.

- Consolidation in the anterior basal segment of right lower lobe suggesting infection.

\section{Task 9}

a) What is the working diagnosis?

b) How should this be managed?

\section{Answer 9}

a) Hydropneumothorax, recurrent empyema, community-acquired pneumonia.

b) The management of recurrent empyema in a patient with a previous surgical intervention for the same condition is challenging. As per current recommendations, the first step to manage empyema is tube thoracostomy [3]. For a patient who has had multiple chest interventions including pleurodesis and decortication for empyema, this is challenging because of previous adhesions and loculations. Chest drain insertion can be facilitated using ultrasound. In addition, pleural cavity drainage and lung re-expansion are often insufficient. If the patient is fit to undergo surgery this should be considered using either VATS or open technique. Reoperation on the grounds of previous empyema surgical treatment can be challenging posing risks for perioperative bleeding and lung injury.

This gentleman had recurrence of his empyema. All surgical options were felt to have been exhausted. He was discharged with conservative management of his empyema, and a 6-week course of co-amoxiclav as advised by microbiology [5].

1 month after discharge, he re-presented to emergency department with shortness of breath, recurrent temperatures, productive cough with green sputum and haemoptysis of $\sim 200 \mathrm{~mL}$. He was haemodynamically stable, his temperature was $36^{\circ} \mathrm{C}$, his heart rate was 75 beats per min, blood pressure was $117 / 63 \mathrm{mmHg}$, respiratory rate was 20 breaths per min and his oxygen saturations were $99 \%$ on room air. His inflammatory markers were stable and his coagulation was within normal limits. He did, however, continue to have haemoptysis of $150 \mathrm{~mL}$ in $24 \mathrm{~h}$.

\section{Task 10}

What initial management and investigations should be performed in a patient presenting with massive haemoptysis?

\section{Task 11}

What are the conservative treatment options for this patient?

\section{Task 12}

What investigatory steps would you consider next? 


\section{Answer 10}

Haemoptysis of over $100 \mathrm{~mL}$ per $24 \mathrm{~h}$ period is considered massive and is a lifethreatening medical emergency. It is usually the result of vessel rupture in an area of chronic inflammation. Initial management is resuscitation, laying the patient on the side of the suspected bleeding and giving oxygen. Initial investigations should include: haemoglobin, platelets, coagulation, group and save blood samples, sputum culture and a chest radiograph. Early involvement of the intensive care team is essential.

\section{Answer 11}

For patients waiting for definitive management or those not suitable for invasive management conservative control of the bleeding can be considered. The following interventions can be considered.

Nebulised adrenaline $(4 \mathrm{~mL}$ of nebulised 1:1000 adrenaline in an emergency), this causes temporary vasoconstriction and is a useful temporising measure. Oral/i.v. tranexamic acid (1-1.5 g 2-3 times a day for 24-48 h) may be used and has been shown to decrease volume and duration of bleeding [6].

i.v. terlipressin ( $1 \mathrm{mg}$ every $4-6 \mathrm{~h}$ for up to $72 \mathrm{~h}$ ) is systemic vasoconstrictor mainly used to control gastrointestinal bleeding, there is limited evidence that it may help control pulmonary haemorrhage, caution should be taken in those with cardiovascular disease [7]. Consider antibiotics/antifungals if it seems likely infection precipitated the bleeding [8].
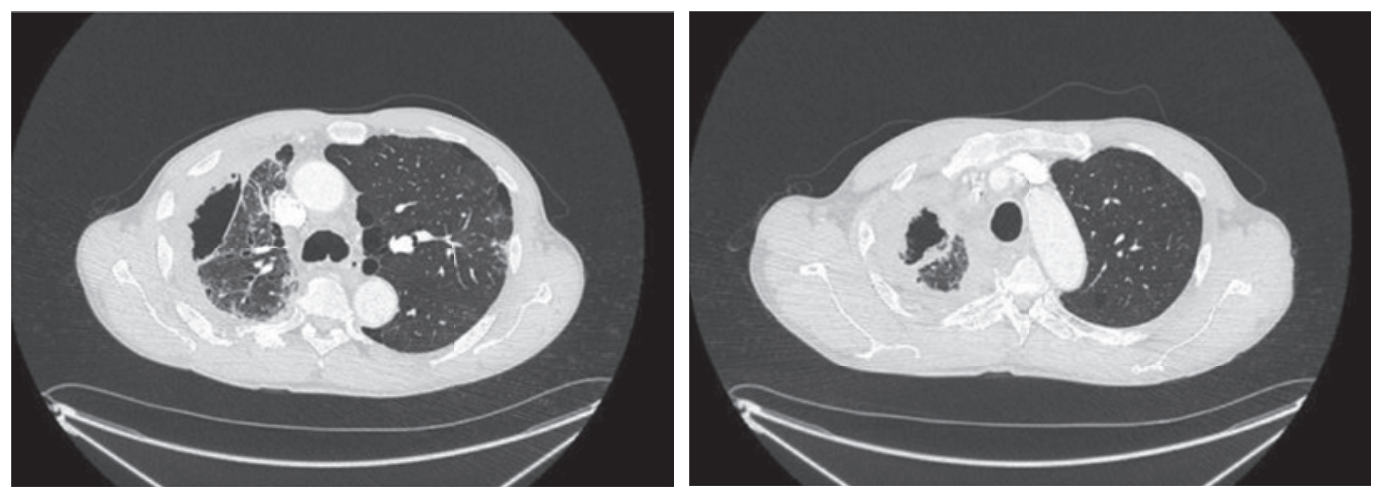

Figure 5 CT scan, August 2017.

\section{Answer 12} the source of bleeding and consider whether embolisation was an option. And if so, to identify which anatomical site would be appropriate for embolisation.

\section{Task 13}

If vessels were identified in figure 5, what are the management options?
CT angiogram scan with contrast to look for 


\section{Answer 13}

Bronchial artery embolisation has emerged as the first line treatment for haemoptysis. CT angiography should be performed as it allows mapping of the bronchial vasculature and helps identify likely sources of bleeding. However, active bleeding is rarely identified on imaging. Catheterisation of the bronchial tree is then undertaken, this is followed by particulate embolisation into the culprit vessel to control the bleeding source. The procedure is minimally invasive and has been shown to be effective in achieving control of bleeding in the short- to medium-term [9]. The most serious complication of bronchial artery embolisation is spinal infarction as in rare cases the left bronchial arteries can contribute to the vascular supply of the anterior spinal artery.

Due to the persistence of symptoms, bronchoscopy was considered to search for the source of endobronchial bleeding. Due to the increased complexity of the case, he was reviewed by a multidisciplinary team including an interventional bronchoscopist, cardiothoracic surgeon and cardiothoracic anaesthetist. During risk assessment, he was deemed to be of high risk for any further intervention due to his previous history of surgical interventions. Bronchoscopy with anaesthetic cover was possible; however, in the event of a torrential haemorrhage access with an open thoracotomy would have been very challenging because of adhesions and underlying trap lung. Lung resection including salvage pneumonectomy was potentially demanding and this could pose delays in case of torrential bleeding.

Taking into account that the haemoptysis settled with conservative management, no further intervention was offered. However, this was considered an opportunity to put into place an advanced care plan for this complicated high-risk patient, in case he re-presented to the emergency department with haemoptysis. There was a consensus among all parties involved that in the case of re-presentation with haemoptysis the only available intervention for him would be embolisation. This was clearly documented in the electronic patient records to ensure future teams are aware and have a clear plan in place.

He was discharged on a prolonged course of oral antibiotics to return for an elective embolisation procedure.

\section{Key learning points}

- Management of acute and chronic pneumothorax

- Management of acute massive haemoptysis

- Management of complicated recurrent empyema and contraindications to surgery

- The importance of multidisciplinary management of complicated high-risk patients

- Advance care plan in place for readmissions

\section{Conflict of interest}

None declared.

\section{References}

1. Havelock T, Teoh R, Laws D, et al. Pleural procedures and thoracic ultrasound: British Thoracic Society pleural disease guideline 2010. Thorax 2010; 65: Suppl., ii61-ii76.

2. Scarci M, Abah U, Solli P, et al. European association for cardiothoracic society expert consensus statement for surgical management of pleural empyema. Eur J Cardiothorac Surg 2015; 48: 642-653

3. Chaturvedi $\mathrm{A}$, Lee $\mathrm{S}$, Klionsky $\mathrm{N}$, et al. Demystifying the persistent pneumothorax: role of imaging. Insights Imaging 2016; 7: 411-429.

4. Campisi P, Voitk AJ. Outpatient treatment of spontaneous pneumothorax in a community hospital using a Heimlich flutter valve: a case series. J Emerg Med 1997; 15: 115-119.

5. British Thoracic Society Pleural Disease Guideline Group. BTS Pleural Disease Guideline 2010. Thorax 2010; 65: Suppl. II, ii1-ii76. www.brit-thoracic.org.uk/ document-library/clinical-information/pleural-disease/ pleural-disease-guidelines-2010/pleural-disease-guideline

6. Moen CA, Burrell A, Dunning J. Does tranexamic acid stop haemoptysis? Interact Cardiovasc Thorac Surgery 2013; 17: 991-994.

7. Fartoukh M, Voiriot G, Hadad S, et al. Severe Haemoptysis. In: Heunks L, Demoule A, Windisch W, eds. Pulmonary Emergencies (ERS Monograph). Sheffield, European Respiratory Society, 2016; pp. 132-150.

8. British National Formulary for medicines. https://bnf.nice. org.uk/drug Date last accessed: March 01, 2018. Date last updated: November, 2017.

9. Chan VL, So LK, Lam JY, et al. Major haemoptysis in Hong Kong: aetiologies, angiographic findings and outcomes of bronchial artery embolisation. Int J Tuberc Lung Dis 2009; 13 : 1167-1173. 